\title{
Neural Correlates of Value, Risk, and Risk Aversion Contributing to Decision Making under Risk
}

\author{
George I. Christopoulos, ${ }^{1}$ Philippe N. Tobler, ${ }^{1}$ Peter Bossaerts, ${ }^{2}$ Raymond J. Dolan, ${ }^{3}$ and Wolfram Schultz ${ }^{1}$ \\ ${ }^{1}$ Department of Physiology, Development, and Neuroscience, University of Cambridge, Cambridge CB2 3DY, United Kingdom, ${ }^{2}$ Ecole Polytechnique \\ Fédérale de Lausanne (Odyssea), CH-1015 Lausanne, Switzerland, and ${ }^{3}$ Wellcome Department of Imaging Neuroscience, Institute of Neurology, \\ London WC1 3BG, United Kingdom
}

Decision making under risk is central to human behavior. Economic decision theory suggests that value, risk, and risk aversion influence choice behavior. Although previous studies identified neural correlates of decision parameters, the contribution of these correlates to actual choices is unknown. In two different experiments, participants chose between risky and safe options. We identified discrete blood oxygen level-dependent (BOLD) correlates of value and risk in the ventral striatum and anterior cingulate, respectively. Notably, increasing inferior frontal gyrus activity to low risk and safe options correlated with higher risk aversion. Importantly, the combination of these BOLD responses effectively decoded the behavioral choice. Striatal value and cingulate risk responses increased the probability of a risky choice, whereas inferior frontal gyrus responses showed the inverse relationship. These findings suggest that the BOLD correlates of decision factors are appropriate for an ideal observer to detect behavioral choices. More generally, these biological data contribute to the validity of the theoretical decision parameters for actual decisions under risk.

\section{Introduction}

Consider somebody selling you a lottery ticket offering $£ 40$ or $\mathfrak{E} 60$, depending on the flip of a coin. You decide to pay up to $\mathfrak{E} 50$ to buy this ticket. Conversely, your friend might consider this ticket as risky and pay a maximum of $\mathfrak{E} 45$. Although both of you face exactly the same average payoff, your reactions are different and vary between risk neutrality (you) and risk avoidance (your friend). Such decisions involving risky options characterize a wide spectrum of human and animal behavior.

Faced with such situations, the agent should accumulate information about the characteristics of the different options and synthesize them to select an alternative. Typically, options with higher expected value (EV) (i.e., the sum of each possible outcome weighted by its probability) are preferred, all other things being equal. However, the introduction of risk influences the subjective value (or utility) attached to a risky option (or gamble). The influence of risk depends on individual attitudes toward risk, with increasing risk aversion reducing the utility of the gamble.

Therefore, decision making is a function of the statistical properties of the options offered (value and risk), with the influence of risk being modulated by the subjective evaluation of the riskiness of the gamble (risk aversion). Risk-averse agents need to

\footnotetext{
Received June 4, 2009; revised July 21, 2009; accepted Aug. 18, 2009.

The study was supported by the Wellcome Trust, the Greek Government Scholarship Foundation, and the Swiss National Science Foundation. P.B. is supported by the Swiss Finance Institute. R.J.D. is supported by a Wellcome Trust program grant. We thank Mathias Pessiglione, Graham Murray, Shunsuke Kobayashi, Krishna Miyapuram, and Nikolaus Weiskopf for helpful discussions.

Correspondence should be addressed to George I. Christopoulos, Laboratory for Interpersonal Decision Neuroscience, Computational Psychiatry Unit, Baylor College of Medicine, One Baylor Plaza, T115, MS:BCM295, Houston, TX77030. E-mail: georgec@cpu.bcm.edu.

DOI:10.1523/JNEUROSCI.2614-09.2009

Copyright $\odot 2009$ Society for Neuroscience $\quad 0270-6474 / 09 / 2912574-10 \$ 15.00 / 0$
}

trade off between value and risk, suggesting that these two parameters are two competing dimensions.

Previous research has begun to identify BOLD responses related to expected value, risk, and risk aversion. Neuroimaging experiments in humans suggest that ventral striatum (VSt) activity increases with EV or its components (magnitude and probability) (Breiter et al., 2001; Knutson et al., 2001, 2005; Abler et al., 2006; Yacubian et al., 2006; Tobler et al., 2007; Rolls et al., 2008). Conversely, activity of the anterior cingulate (ACC) has been associated with the volatility of reward environment (Behrens et al., 2007) and the variability of expected outcomes (Critchley et al., 2001; Brown and Braver, 2005, 2008; Kuhnen and Knutson, 2005). Dorsal ACC (dACC) has been related to directing action selection for uncertain rewards (Hampton and O'Doherty, 2007). Although neural correlates of EV and risk have been extensively studied, the neural basis of attitudes toward risk in a choice situation is less well described (Tobler et al., 2007). Yet, the right dorsolateral prefrontal cortex (DLPFC) has been implicated in the modification of risk attitudes (Knoch et al., 2006; Fecteau et al., 2007).

However, it is less well known whether these parameterrelated BOLD signals merely reflect the characteristics of the choice situation or actually carry information that combines to contribute to the choice process. We hypothesized that BOLD signals in different brain structures reflecting key decision parameters can combine in a way that allows an ideal observer to detect the nature (risky or safe) of the behavioral choice during risky decision making. To investigate this hypothesis, in two different experimental paradigms, we identified BOLD responses preferentially encoding value [magnitude (first experiment) and/or expected value (second experiment)] risk and risk aversion. Subsequently, we used these parameter-specific responses to test the 
A

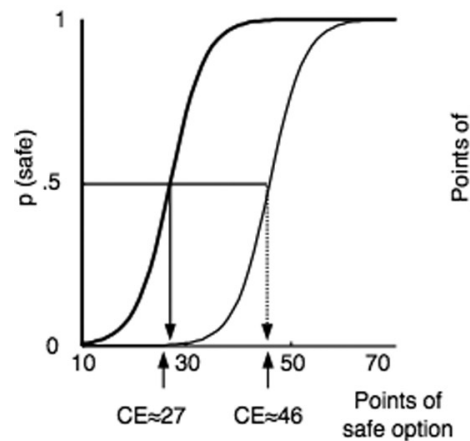

C

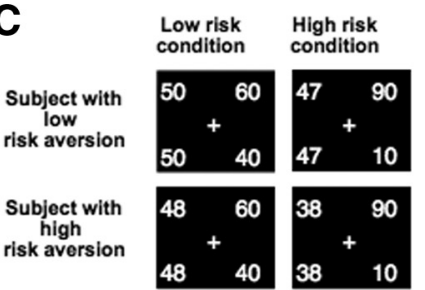

E

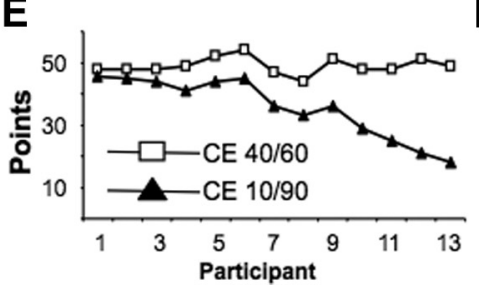

B

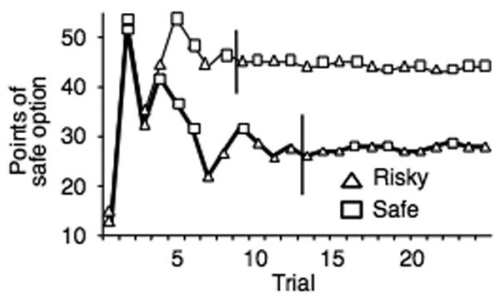

D $\begin{gathered}\text { Expected } \\ \text { value }\end{gathered}$

Risk

Utility
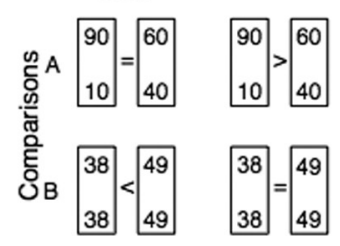

$\begin{array}{ll}90 \\ 10\end{array}<\begin{aligned} & 60 \\ & 40\end{aligned}$

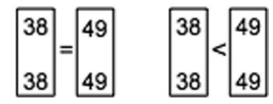

$\mathbf{F}$

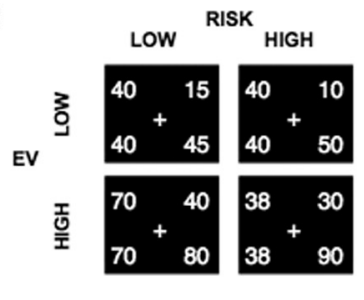

Figure 1. Task and behavioral results. $A$, Psychophysical definition of certainty equivalent. The CE of a gamble is the amount for which an agent is indifferent between receiving it for sure and opting for the gamble. This definition implies that the probability of choosing the CE instead of the gamble is $p=0.5$. Examples show probability distributions of safe choices as a function of safe amounts for two participants with different degrees of risk aversion (thick line for stronger risk aversion with lower (E). $\boldsymbol{B}$, Iterative determination of CE. In each trial, participants chose between a safe and a risky option. The staircase method (PEST procedure) iteratively adjusted the safe option in consecutive trials to approximate choice indifference between the two options. Lines show data from two participants with different CEs (thicker line represents higher risk aversion). The shape of each dot illustrates safe and risky choices. Vertical lines indicate good approximation of indifference values and mark onset of scanning. C, Choice options as presented to participants (first experiment). Participants chose between either a safe option or one of two gambles with two equiprobable outcomes (40/60 and 10/90, respectively). Each screen shows a safe (left) and a risky option, the safe value being set to choice indifference. The first row represents the choice set a less risk-averse participant faced, whereas the second row the choice set of a very risk-averse participant. The first column represents the low risk condition (choices involving the low risk gamble), whereas the second column represents the high risk condition (choices involving the high risk gamble). D, Differential assessment of key decision parameters: EV, risk (as increase in spread), and utility. Each comparison serves to identify differences in two of these parameters. Comparison A tests differences in risk and utility but not EV; comparison B tests $\mathrm{EV}$ and utility, controlling for risk. $E$, $\mathrm{CE}$ of participants. CEs of individual participants for the two gambles (40/60 and 10/90) are displayed according to increasing risk aversion. Lower CEs, and larger differences between $C E s$ for the two gambles, indicate increasing risk aversion. $F$, Choice options as presented to participants (second experiment). Participants again chose between a safe and an even-chance gamble. This time, four gambles were used: the first two (offering $£ 10$ or $£ 50$ and $£ 15$ or $£ 45$, respectively) had expected value of $£ 30$, whereas the other two (offering $£ 40$ or $£ 80$ and $£ 30$ or $£ 90$, respectively) had an expected value of $£ 60$. For gambles with the same expected value, one was riskier than the other. Importantly, safe alternatives were not set to indifference level but took semi-random values.

preserving increase in the spread of outcomes. This definition is analogous to risk measures such as SD and variance in skewness free distributions with the other moments kept constant (e.g., expected value/mean). Notice that the gambles used in this study also coincide with other definitions of risk (coefficient of variation) (Weber et al., 2004; McCoy and Platt, 2005).

\section{Subjective risk (risk aversion)}

Conversely, risk aversion is subjective. The degree of risk aversion can be behaviorally demonstrated within a psychophysical framework by identifying the safe amount for which the agent is indifferent in choices against a risky outcome (Luce, 2000) (Fig. 1A). This indifference amount, or certainty equivalent (CE), precisely reflects the value attached to the risky option and allows for comparisons between different options and their expressions across individuals. For instance, a risk-neutral agent will attach the same CE to both 40/60 and 10/90 gambles. On the contrary, a risk-averse decision maker will be affected by the increase of risk from the $40 / 60$ to the $10 / 90$ gamble and will lower her CE for the riskier option. Hence, the difference between the certainty equivalents of each gamble reflects the degree of risk aversion of the agent.

\section{Participants}

All participants were right-handed, had normal or corrected-to-normal vision, and were screened to exclude those with a previous history of neurological or psychiatric disease. All gave informed written consent. The Local Research Ethics Committee (National Hospital for Neurology and Neurosurgery and the Institute of Neurology Joint Research Ethics Committee) approved the study. In the first experiment, three risk-seeking participants were excluded. One more participant was excluded (and not scanned) because she or he violated monotonicity. These participants were not scanned. Restriction to risk-averse agents was done to ensure homogeneity of data and straightforward evaluation of results. In the second experiment, there were no risk-attitude-related exclusion criteria; still all participants more or less exhibited risk aversion (see Results), which is in accordance with numerous studies suggesting that persons predominantly exhibit risk-averse preferences (Binswanger, 1980; Holt and Laury, 2002; Dohmen et al., 2005). extent to which they could detect the choice behavior and decode their contribution to the probability of a risky or a safe choice.

\section{Materials and Methods \\ Definitions}

Objective risk

Under certain conditions, risk can be objectively defined. Rothschild and Stiglitz (1970) provide a formal, minimal definition of risk and characterize it as the spread of outcomes, with the condition that the EV of options is preserved. For example, a gamble equiprobably $(p=0.5)$ offering 10 or 90 points (10/90) is riskier than a gamble equiprobably offering 40/60. Probabilities and EV are the same, but risk is different. In this study, we follow the Rothschild-Stiglitz definition of risk as a mean-

\section{Experimental tasks and behavior}

\section{First experiment}

All participants made repeated choices between a risky ("gamble") and a safe alternative, offering a single amount with certainty. In the first experiment ( $n=13$; mean age of 24.5 years, five females), two gambles were offered, one resulting in either 40 or 60 points (low risk gamble) and one, riskier, resulting in either 10 or 90 points (high risk gamble), in which each outcome had an equal probability ( $p=0.5$ ) of occurrence (Fig. $1 C$ ). Participants were paid according to the cumulative total amount of points (converted to real money) they acquired during the experiment.

For the first experiment, we adjusted the value of the safe options according to the risk preferences of each participant. We did this to ensure that, within each choice set, the alternatives had the same utility [it 
should be underlined that the concept of utility as used here refers to decision utility, i.e., an ordering representing preference, and not to experienced utility, which refers to the pleasure derived from the consumption of an outcome (Kahneman et al., 1997)]. To achieve this, we approximated, before scanning, the CEs for both gambles for each participant, using a staircase method (Fig. $1 B$ ) [parameter estimation by sequential testing (PEST)] (Luce, 2000) (supplemental data, available at www.jneurosci.org as supplemental material). During scanning (Fig. $1 B$, trials after the vertical lines), the safe alternatives were initially set to the corresponding, previously determined, approximated CE; consequently, their value was updated to accommodate temporal variations in risk aversion. This method ensured an approximately equal number of risky and safe choices [by $\chi^{2}$ test, all comparisons were nonsignificant (NS)], which reflected indifference between the values of the safe alternative and the risky gamble. In addition, choices between risky and safe options were independent of the previous choices and did not constitute simple alternations $(p>0.1)$. Therefore, by the overt behavioral preferences shown ("revealed preferences"), for each participant the utilities of a gamble and its safe alternative were the same, because both were equally preferred.

Choice trials were randomly interspersed with no-choice trials. In each choice trial, participants were presented, on a computer monitor, with two alternatives (Fig. 1D) (supplemental data, available at www.jneurosci.org as supplemental material), randomly positioned to the left and right of an ocular fixation cross (evaluation phase). Participants had always to choose between a risky and a safe option. Two risky options with equiprobable outcomes were used (40/60 and 10/90). After $5.5 \mathrm{~s}$, the fixation cross was circled ("go" signal), signaling that the participant should press a button to indicate her choice. If the participant failed to respond within $600 \mathrm{~ms}$, an error message appeared. In correct trials, the circled cross remained on the screen until $1000 \mathrm{~ms}$ had elapsed; subsequently, the choice was framed for a random period with an average of $4 \mathrm{~s}$ ( $2 \mathrm{~s}$ fixed plus $2 \mathrm{~s}$ variable according to a exponential distribution truncated at $15 \mathrm{~s}$ ), allowing temporal decorrelation (via jittering) (Dale, 1999) between choice and outcome phase. Subsequently, the outcome of the choice was shown for $1 \mathrm{~s}$. A cross, to which participants had to fixate, appeared for the same random period, indicating the onset of a new trial. Fixation was added to allow temporal decorrelation of the outcome phase with the presentation of options in the next trial. No-choice trials had exactly the same sequence of screens as choice trials, with the exception that, during the presentation of options, a small arrow placed next to the fixation cross indicated what the choice should be (left or right). The participant had to press the corresponding button; otherwise, an error screen was shown.

Structure of experiment. The actual experiment started with an "estimation session," during the acquisition of structural images. During the estimation session, we approximated the CE of each participant, for both gambles. This was followed by three sessions of the task ("main sessions"), during which functional scans were acquired.

Main (scanned) sessions. After the estimation session, each participant played three main sessions, during which functional images were acquired. During each session, the participant faced $20 \times 2$ (choice/nochoice $) \times 2$ (high/low risk $)=80$ trials minus the errors (average errors per participant was 4.7 ).

To ensure an approximately equal number of safe and risky choices, denoting that the risky and safe alternatives have the same utility, the safe alternative was updated according to a PEST-like algorithm that took into account previous choices (supplemental data, available at www. jneurosci.org as supplemental material).

The payment method limited wealth effects and diversification strategies (supplemental data, available at www.jneurosci.org as supplemental material).

Estimating certainty equivalent. Because of the updating algorithm, the values of the safe alternative during scanning reflected indifference with the risky option, adjusted for temporal variations in risk aversion. For each participant and gamble, the median of these values was set to be the corresponding CE.

\section{Second experiment}

Structure of experiment. In experiment 2, a separate group of 14 participants had again to choose between a risky and a safe option. The main changes were as follows: (1) the outcome of each choice was not shown, ensuring that decision-related responses were not influenced by outcome-related responses, and choices were not influenced by the history of previous outcomes; (2) only one choice obtained at the end of the experiment (i.e., participants did not accumulate points after each choice; they were told that they will make a series of decisions but only one, randomly selected choice will determine their reimbursement) to remove any wealth effects; (3) the safe alternatives were not set to indifference level, which allowed testing that the responses were independent of the value of the safe alternative; (4) no-choice trials were not used (increasing power); (5) gambles represented real money rather than points (increasing participant involvement); and (6) risky gambles were studied at two different levels of mean gain, allowing additional testing of value processing.

Four even-chance gambles were offered $(\mathfrak{E} 15 / \mathfrak{£} 45, \mathfrak{E} 10 / \mathfrak{E} 50, \mathfrak{E} 40 / \mathfrak{E} 80$,

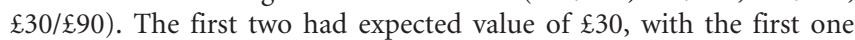

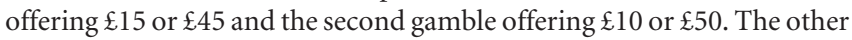
pair of gambles had an expected value of $£ 60$, with the first one offering $\mathfrak{E} 40$ or $\mathfrak{1} 80$ and the second one offering $\mathfrak{E} 30$ or $\mathfrak{1} 90$. Therefore, within each pair, one gamble was riskier than the other. The trial structure was exactly the same as in the first experiment, with the exception that no outcome was shown. The second experiment comprised two sessions. Each session consisted of 20 trials per gamble.

Estimating certainty equivalent. The CE was estimated as the frequencyweighted average of the values of the safe alternative for which participants at some point during the experiment chose both the risky and safe option (supplemental data, available at www.jneurosci.org as supplemental material).

\section{Measuring risk aversion: certainty equivalents}

We identified the risk aversion of each participant using the CEs. The difference between the CEs of two gambles with the same EV $\left(\mathrm{CE}_{\text {low risk gamble }}-\mathrm{CE}_{\text {high risk gamble }}\right)$ reflects risk aversion. Less riskaverse participants [with low difference between the CEs of the two gambles (Fig. 1E, left side)] (for results of the second experiment, see supplemental data, available at www.jneurosci.org as supplemental material) perceive the increase in risk as less important compared with more risk-averse participants (Fig. $1 E$, right side), who perceive the risk manipulation as a significant escalation of risk. As a result, riskaverse participants had lower safe alternatives for the high risk gamble (Fig. 1C). A larger difference between the two CEs indicates higher risk aversion.

\section{Data analysis: imaging}

Image acquisition and preprocessing parameters are described in the supplemental data (available at www.jneurosci.org as supplemental material). For each participant, all instances of a particular event type were modeled through convolution with a canonical hemodynamic response function (and its temporal and dispersion derivatives). For the first experiment (analyzed with SPM2) (supplemental data, available at www. jneurosci.org as supplemental material), in the first-level analysis, two main events were included in the same model: presentation of gambles and presentation of outcome. Both events had eight different conditions, forming a $2 \times 2 \times 2$ design: 2 (choice or no-choice) $\times 2$ (high or low risk gamble condition $) \times 2$ (safe or risky choice). As a result, 16 regressors were entered for each participant. For the second experiment (analyzed with SPM5), one main event was included (presentation of options); the event had 4 (four gambles) $\times 2$ (risky or safe choice) $=8$ conditions. Errors were modeled as a different regressor. Movement parameters and errors were modeled as covariates of no interest.

We tested for different temporal profiles of BOLD response [phasic and sustained, with duration equal to the time until the go signal appeared $(5.5 \mathrm{~s})$ ]. For each participant, two different models were constructed to evaluate phasic (event-related design) and sustained response (5.5 s epoch-based design) to the onset of options. We used a participantspecific, fixed-effects model for each event type. Parameters estimates for 
each regressor were calculated for each voxel (Friston et al., 1995). Contrast images were constructed, demonstrating the size of the certain effect at each voxel. Subsequently, these data were entered into a second-order, random-effects analysis (Friston et al., 1999). At that level, contrast images were entered into one-sample $t$ tests, simple regressions, or ANOVAs. Nonsphericity correction [as implemented in SPM2 and SPM5 and described by Glaser and Friston (2003)] was used at ANOVA analyses.

Throughout, we used whole-brain or small-volume correction (svc) for multiple comparisons controlled at $p<0.05$ (family-wise error). We used small-volume correction with family-wise error controlled at $p<$ 0.05 for the analysis for value and risk. Because previous studies (described in Introduction) have implicated VSt and cingulate cortex for EV and risk encoding, they were used as a priori anatomical regions of interest (ROIs). Despite that many studies (Paulus et al., 2003; Kuhnen and Knutson, 2005; De Martino et al., 2006; Huettel et al., 2006) have examined the BOLD responses of risk-averse choices, none of them had incorporated in their analysis the model-free subjective estimation of the riskiness of the gamble. Therefore, selecting an ROI (such as insula) would have been unjustifiable; as a result, we used whole-brain correction for the related analysis. Therefore, right DLPFC activity was corrected for the whole brain. Ventral striatum ROI was hand drawn in MRIcro (Rorden and Brett, 2000) according to the anatomical description by Martinez et al. (2003), as used previously (Murray et al., 2008), adjusted to the anatomical specificities of our sample. The cingulate ROI included anterior and posterior cingulate and was constructed using the Pickatlas Toolbox (Maldjian et al., 1997). Reported voxels conform to MNI (Montreal Neurological Institute) coordinate space, with the right side of the image corresponding to the right side of the brain. The most significant voxel (peak voxel) within the cluster of activation is reported.

\section{Results}

\section{Behavior: risk aversion}

The mean CEs for the first experiment were 48.9 points (low risk gamble) and 35.3 points (high risk gamble). For the second experiment, the mean CEs were $\mathfrak{E} 25.7$ (gamble offering $\mathfrak{E} 15 / \mathfrak{E} 45$ ), $\mathfrak{E} 23$ (gamble $\mathfrak{1} 10 / \mathfrak{E} 50$ ), $\mathfrak{E} 53.8$ (gamble $\mathfrak{E} 40 / \mathfrak{E} 80$ ), and $\mathfrak{E} 48.1$ (gam-

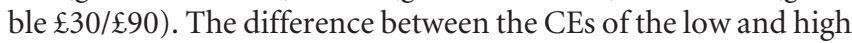
risk gamble was used to measure risk aversion. For the first experiment, risk aversion coefficients ranged from 2.5 to 31 (mean of 13.57), with higher values implying higher risk aversion. For the second experiment with a comparable risk assessment, risk aversion coefficients ranged between $-\mathfrak{E} 0.87$ and $\mathfrak{E} 8$ (mean of $\mathfrak{E} 2.66$ ) for the low EV gambles ( $\mathfrak{1} 15 / \mathfrak{k} 45$ and $\mathfrak{E} 10 / \mathfrak{£} 50)$ and between $-\mathfrak{E} 1.5$ and $\mathfrak{E} 12.08$ (mean of 5.69) for the high EV gambles

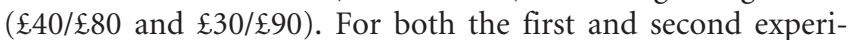
ments, the values of the CEs of the low and high risk gambles were statistically different from each other [first experiment, $p<0.001$ (paired $t$ test); second experiment, $p<0.001$ (high mean gambles) and $p<0.005$ (low mean gambles)].

\section{Neuronal correlates of decision parameters}

We tested the BOLD responses to the onset of the stimuli (presentation of the risky and safe alternative).

\section{Magnitude/expected value coding}

First experiment. We identified value coding in the brain. In our paradigms, the expected value changes while keeping the probabilities constant, according to the Rothschild-Stiglitz definition of risk. In the first experiment, the difference between the monetary values of the two safe alternatives varies across participants (Fig. $1 F$ ). Therefore, an area encoding magnitude should be sensitive to this variability. We measured the differential BOLD response preceding the choice of the safe alternatives [comparison $\mathrm{B}$ (Fig. 1D): Activity low risk safe $_{\text {- Activity }}$ high risk safe] and corre- lated it with the value difference between the two CEs. The difference between the values of the CE positively correlated with increasing differential response of VSt (Fig. $2 A, B 1$, solid line) (peak at $-14 / 6 /-2 ; R^{2}=0.68 ; p<0.01$, small-volume correction) (Worsley et al., 1996), suggesting that this area is sensitive to magnitude. A similar result was found in the no-choice trials (Fig. $2 B 2$, solid line) $\left(-20 / 8 /-8 ; R^{2}=0.70 ; p<0.01\right.$, svc $)$.

We also performed a paired $t$ test comparing BOLD responses to the safe alternatives. When all participants are included, the striatal response does not survive correction. However, this apparently negative result could be misleading: it is most likely a result of the fact that, for participants that are close to risk neutrality, the difference in the values of the safe alternatives between the two conditions of interest is very small (i.e., $\leq 3$ units); consequently, the associated small difference in BOLD response may add primarily noise to the $t$ test (but not to the correlation). In other words, given that the difference in value for the almost-riskneutral agents is small, it should also be expected that the difference between the BOLD responses corresponding to these safe alternatives should also be small.

In agreement with this reasoning, if we exclude the two almost-risk-neutral participants, then a significant striatal response differentiating between the values of the safe alternatives appears also in the $t$ test, even though the sample is smaller $(p<$ 0.05 , svc; $4 / 12 /-8$ and $-12 / 20 /-4)$. This response is bilateral.

Note that the two excluded participants are not handpicked. They are the participants that have a very low difference between the safe alternatives they face; indeed, for these two participants, the difference between safe alternatives was $>1$ SD away from (i.e., smaller than) the mean difference of the safe alternatives of the group.

Interestingly, exactly the same happens in the no-choice trials: including all participants, the striatal response seems to be unable to differentiate between the different magnitudes. Again, excluding exactly the same participants, the striatal response significantly differentiates between the two conditions $(p<0.05$, svc; 4/10/2).

Together, these results suggest that the striatum codes value in both choice and no-choice situations, as long as differences in value are clearly present in the behavior.

Control for utility encoding. The experimental design also allowed controlling for utility encoding: the striatal response could actually be interpreted as representing utility (or pure preferences), because larger differences between the values of the safe alternatives represent larger differences in utility as well. We controlled for this confound by using the comparison between the two risky options (comparison A: Activity ${ }_{\text {high risk }}$ gamble - Activity $_{\text {low risk gamble }}$ ). Note that gambles have the same utility with their corresponding safe alternatives. If striatum encodes utility, then the differential activity between the two risky options should also correlate with the differences between the two safe alternatives. We found that striatal activity did not change with respect to utility differences (Fig. 2B1,B2, dotted lines) $\left(R^{2}=0.0\right.$, NS) between the low and the high risk gamble. An analysis of the interaction effects, comparing the slopes of the two regression lines, was significant for both the choice and no-choice trials $(p<0.05)$.

Second experiment. We used the data from the second experiment and compared the activity between the high and low mean gambles to test whether striatum activity changes with $\mathrm{EV}$. We first compared the safe conditions (in which the subsequent choice was a safe alternative) with different magnitudes (high vs low values of safe choice); the comparison confirmed striatal sen- 
A

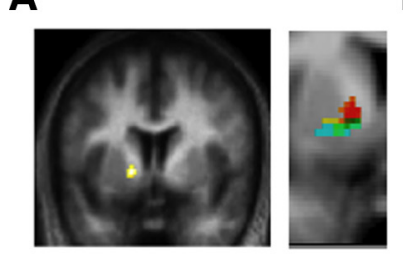

C
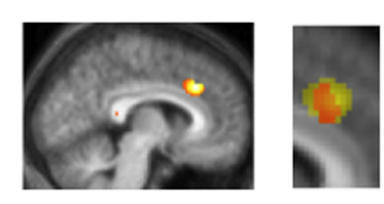

B1

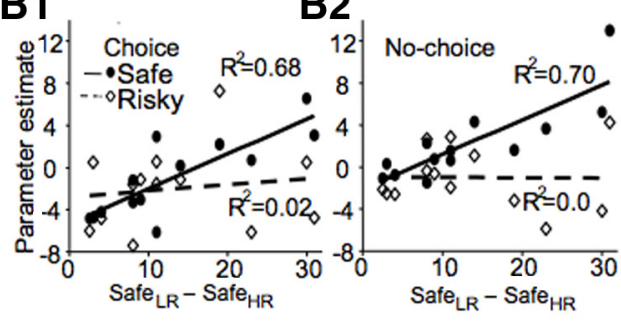

D1

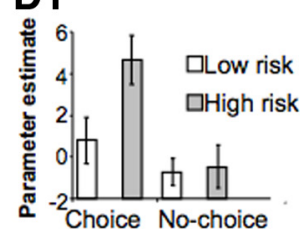

D2

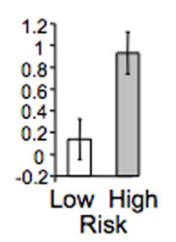

Figure 2. Brain activity related to value and risk. All BOLD responses presented were modeled on presentation of the stimuli (options) and are estimated by the related regression slope parameter estimates $(\beta)$. $\boldsymbol{A}$, Value coding by VSt. Response location in VSt sensitive to magnitude/EV differences ( $p<0.05$, small volume correction, displayed at $p=0.01)$. Red, First experiment, comparing safe alternatives, choice situation; yellow, first experiment, same in no-choice situation; green, second experiment, comparing safe choices having different magnitude; blue, second experiment, comparing risky choices having different EV. Darkest voxels reflect common activation areas. B, Quantitative value coding by ventral striatum. $\boldsymbol{B}$, Increasing difference in the magnitude of the safe alternatives of each gamble ( $x$-axis) correlates with the differential VSt response to the choice of these alternatives ( $y$-axis) (solid line; $R^{2}=0.68 ; p=0.0005$ ). This signal does not change when we compare high risk (HR) and low risk (LR) gambles (comparison A in Fig. 1, signaling either risk or utility) (dotted line). B2, The same area shows a similar activation pattern in no-choice trials. $\boldsymbol{B} 3, \mathbf{B}$, In addition, a neighboring voxel (peak at $-22 / 6 / 8$ ) distinguishes between high and low expected value in the second experiment. $\boldsymbol{B} 4$ is essentially the same as $\boldsymbol{B}$, with the exception that we now compare the two risky options with different expected values, whereas in the first experiment, we compared safe options with different magnitudes. $C$, Risk coding by dACC. Comparing activity emerging from a choice of the high risk option to activity related to a choice of the low risk one, risk-sensitive areas were identified. This comparison reached significance in $\mathrm{dACC}(p<0.05$, displayed at $p=0.01)$. This signal also does not covary with risk attitudes or the utility of each option. Red, First experiment, comparing high and low risk gambles, choice condition; yellow, second experiment, comparing high and low risk gambles. D, Quantitative coding of risk by $\mathrm{dACC}$. $\mathrm{dACC}$ shows higher response for the high risk gamble than to the low risk option. $\mathbf{D 1}$, An interaction effects analysis suggested that this sensitivity of dACC to the high risk occurs only in choice trials and not in no-choice trials $(p<0.05)$. Error bars represent SEM. D2, The same area showed increasing response to high risk compared with low risk in the second experiment, in which the safe alternatives are not set to indifference level. This suggests that the dACC response to higher risk is not attributable to the lower value of the alternative offer (which is the case in the first experiment).

sitivity to magnitude (Fig. 2 B3) (peak at $-12 / 12 /-8 ; p<0.05$, $\mathrm{svc}$ ). In addition, we also compared the BOLD responses preceding a choice of a high versus a low EV gamble (i.e., including only the occasion in which the choice is risky), and we found a sustained response to the gambles with higher $\mathrm{EV}$, again in striatum (Fig. 2B4) (peak at $12 / 6 /-8 ; p<0.05$, svc).

\section{Risk coding}

First experiment. To test risk coding, we compared the BOLD response preceding choices of the high risk gamble with the response preceding low risk gamble choices. dACC (Fig. 2C) (peak at $8 / 30 / 34 ; p<0.05$ ) showed higher BOLD response when the subsequent choice was the high risk gamble compared with when the choice was the low risk gamble). Interestingly, such an activity differentiating between high and low risk trials was not found in no-choice trials, potentially signifying that the dACC response is mainly choice specific (interaction analysis, $p<0.05$ ).

Control for magnitude or utility coding. We also tested whether the dACC signal is independent from magnitude or utility variations. To control for these, we compared the dACC response to the safe alternatives of each gamble (Activity low risk safe - Activity $y_{\text {high risk safe }}$ ). On average, the value of the low risk safe alternative (mean of 48.92) is higher than the value of the high risk safe alternative (mean of 35.34). If the ACC signal was in fact encoding either EV or utility, then it should differentiate between the two safe alternatives. We observed a significant interaction showing that, whereas dACC differentiates between the two risky options, it shows no differential activity between the corresponding safe alternatives ( $p<0.05$, whole-brain correction). Moreover, dACC activity does not covary with risk aversion $\left(R^{2}=0.05, \mathrm{NS}\right)$.
This potentially suggests that dACC encodes risk in an objective manner, regardless of magnitude, utility, or the subjective evaluation of the riskiness of the gambles (risk aversion).

Second experiment: risk coding with control for fictive/regret signals. In the first experiment, because of the indifference level setting, the safe alternative of the high risk gamble is lower compared with the alternative of the low risk gamble. The difference in the BOLD response between high and low risk gambles might therefore be attributable to the fact that their safe alternatives differ in magnitude (Loomes and Sugden, 1982; Lohrenz et al., 2007). Accordingly, this difference could actually reflect an inverse coding of the foregone safe amount. On the contrary, in the second experiment, the safe alternatives are approximately the same across gambles with the same $\mathrm{EV}$, because they were not adjusted to indifference levels; therefore, the comparison between high and low risk gambles does not suffer from the possibility that the safe alternatives are also different (as is the case in the first experiment). To control for this possibility, we used data from the second experimental paradigm, in which the offered safe alternatives are not set to indifference level and are approximately the same across participants and gambles with the same EV. Again, the risk encoding function of dACC was found when we compared

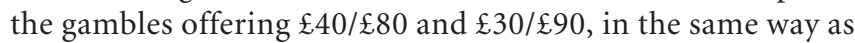
in the first experiment. Dorsal ACC was sensitive to higher risk (Fig. 2D2) (peak at 16/22/28; $p<0.05$, svc). As in the first experiment, this difference weakly and insignificantly correlated with risk attitudes, expressed as the difference between the CEs $\left(R^{2}=0.16, \mathrm{NS}\right)$. 

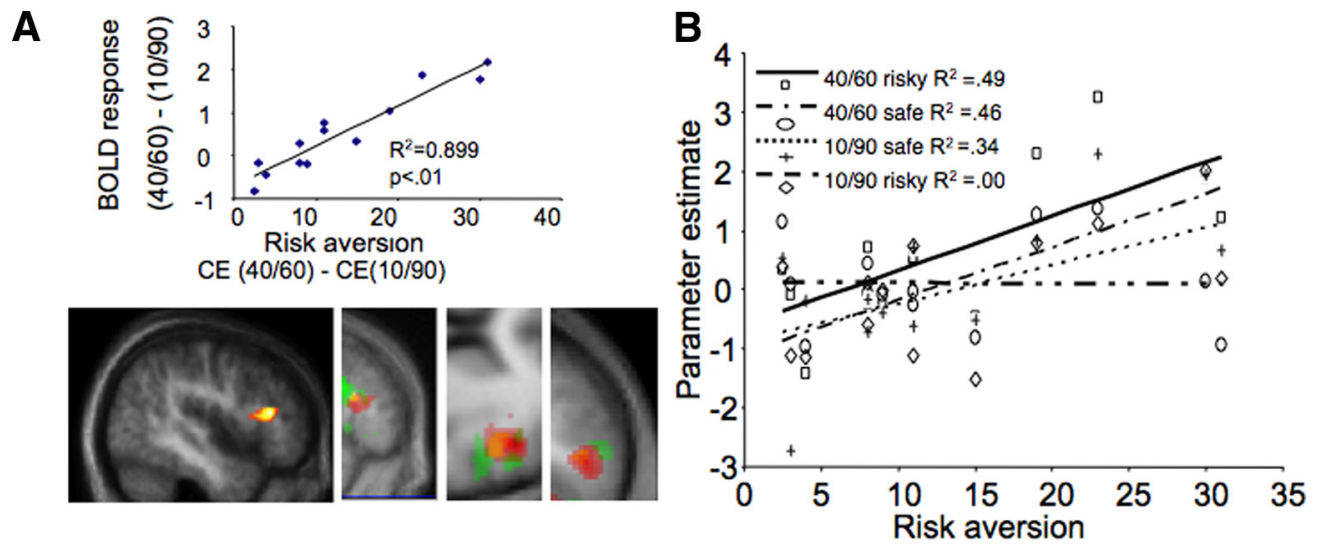

Figure 3. Modulation of IFG activity by risk aversion. $A$, Increased differential IFG activity with risk aversion. $y$-axis represents the difference of the IFG parameter estimate of the BOLD response preceding a choice of the low risk gamble minus the corresponding IFG parameter preceding a choice of the high risk gamble. $x$-axis represents risk aversion of each participant, as measured by the monetary difference between the $\mathrm{CE}$ of the two gambles ( $\left(\mathrm{E}_{\text {low risk gamble }}-\mathrm{CE}_{\text {high risk gamble }}\right.$ ). The more risk averse the participant, the larger the difference in $\mathrm{B} 0 \mathrm{LD}$ response in IFG $(p<0.05$, whole-brain correction). The first image is from the first experiment. The next three images depict sagittal, axial, and coronal planes showing the common right DLPFC activated voxels for the risk-attitude-related contrast. Red, First experiment, choice condition; yellow, first experiment, no-choice condition; green, second experiment. $\boldsymbol{B}$, Correlation of BOLD response in IFG to safe and low risk gambles with individual risk aversion. The IFG response slope increases with gambles of decreasing risk, thus providing better discrimination of lower risks in risk-averse participants. This selective coding of a "safety signal" for more risk-averse participants is verified by the similar (increasing) activity of the same voxel as a response to safe choices. In contrast, activity related to a choice of the high risk option does not correlate with individual risk aversion. The $R^{2}$ values for each regression line are as follows: low risk gamble, 0.49 ; low risk safe, 0.46 ; high risk safe, 0.34 ; high risk gamble, 0.00 .

Differential response to risky options according to risk aversion First experiment. The fact that participants attached different CEs to each gamble suggests that the increase in risk (from the low risk to the high risk gamble) was perceived differently by each individual. This subjective evaluation of risk is reflected in the difference between the CEs. In both experiments, participants demonstrated sufficient variability of risk attitudes, enabling us to study the corresponding differences in BOLD response (Friston et al., 1999). To determine whether these individual differences in behavioral responses to risk are reflected in brain activity, we correlated the difference in the brain response to the two gambles [comparison A (Fig. 1D): Activity ${ }_{\text {low risk gamble }}-$ Activity $_{\text {high risk }}$ gamble] with risk aversion [defined as the difference between the values of the two $\left.\mathrm{CEs}\left(\mathrm{CE}_{\text {low risk gamble }}-\mathrm{CE}_{\text {high risk gamble }}\right)\right]$. A strong correlation $\left(R^{2}=0.89 ; p<0.01\right.$, whole-brain corrected) (Fig. 3A) was evident in the inferior frontal gyrus (IFG) (48/32/ 14) (Fig. $3 A$ ): increasing risk aversion provoked better differentiation between the BOLD responses of the two gambles, reflecting the behavioral difference in the CEs. This result is based on the increased IFG activity preceding a choice of the low risk gamble in correlation with risk aversion (Fig. $3 B$ ); on the contrary, activity for the high risk gamble remains unchanged (again with respect to risk aversion). A similar, yet less strong, result was also found in the no-choice trials (peak at 48/26/14; $R^{2}=0.77$; $p<0.05$, svc). IFG activity differentiated between high and low risk according to risk aversion, in a similar way to choice trials.

There were no other areas surviving whole-brain correction. Nevertheless, given that insula has been implicated in risk-averse choices, we tested whether the BOLD response of this area correlates with risk aversion, using an ROI analysis. In the first experiment, there is a nonsignificant trend of anterior insula correlating with risk attitudes. However, this activation does not survive at all in the second experiment. We are therefore forced to reject the hypothesis that insula BOLD responses correlate with risk attitudes.

Second experiment. In the second experiment, we correlated

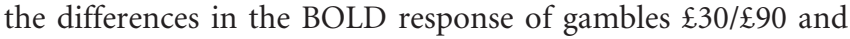
$\mathfrak{E} 40 / \mathfrak{E} 80$ with risk aversion. Again, right IFG BOLD response (peak at 52/14/22; $p<0.05$, false discovery rate, svc) correlated
Table 1. Logistic regression parameters detecting decisions from ventral striatum, $\mathrm{dACC}$, and IFG BOLD response

\begin{tabular}{lcllll}
\hline Variables & Coefficients $(\beta)$ & SE & Wald statistic & $p$ & Odds ratio $[\operatorname{Exp}(B)]$ \\
\hline VSt & 1.161 & 0.152 & 58.131 & 0.000 & 3.195 \\
dACC & 0.966 & 0.136 & 50.528 & 0.000 & 2.626 \\
IFG & -0.326 & 0.158 & 4.276 & 0.039 & 0.722 \\
Constant & -0.141 & 0.055 & 6.549 & 0.010 & 0.869 \\
\hline
\end{tabular}

with risk aversion, in a similar manner as in the first experiment (positive correlation in parallel with increasing risk aversion) (supplemental data, available at www.jneurosci.org as supplemental material). Hence, these results suggest that IFG BOLD response increases with lower-risk gambles, and this increase is more pronounced in risk-averse agents.

\section{Decoding the behavioral choice by BOLD response}

We next sought to identify whether neuronal signals solely reflect decision-making parameters or, are, in addition, relevant to actual choice behavior. Our analysis so far identified three different structures preferentially processing basic decision parameters: VSt, dACC, and IFG, reflecting magnitude, risk, and risk aversion, respectively.

Using a similar methodology with previous studies (Kuhnen and Knutson, 2005; Knutson et al., 2007), we used binary logistic regression (Hosmer and Lemeshow, 1989) to determine whether the combination of BOLD responses correlates with a risky or safe choice and also to elucidate the potential contribution of each structure to decision making. We used three variables (trialby-trial activity of VSt, dACC, and IFG) and the actual choice made on each trial (risky or safe) as the dependent variable.

Overall coefficients of the logistic model related to activity of each structure were significant (Table 1), suggesting that all three structures contributed to the choice. Importantly, the overall logistic regression coefficients allowed us to clarify the exact role of each structure. Logistic regression coefficients were positive for VSt and dACC activity $\left(B_{\mathrm{VSt}}=1.161, B_{\mathrm{dACC}}=0.966 ; p<0.05\right)$, whereas IFG had a negative coefficient $\left(B_{\mathrm{IFG}}=-0.326 ; p<\right.$ $0.05)$. A similar result was obtained in the second experiment 
(supplemental data, available at www.jneurosci.org as supplemental material). This indicates that increasing activity of VSt and ACC increases the probability of a risky choice, whereas the IFG activity pattern does the opposite (Fig. 4C). To further investigate the contribution of IFG activity to choice behavior, we identified how the model-based probabilities of a risky choice change with different levels of IFG activity, in relation to the activity of VSt and dACC. Increasing IFG activity moves the psychophysical function toward the right (Fig. 4D). Thus, the overall logistic regression model suggests that, given an increase in IFG activity, increased VSt and ACC activity is required to obtain the same probability of a risky choice.

Above, we presented the logistic regression results from a fixed-effects analysis to evaluate the overall contribution of the three structures, independent of variability in between-subjects task characteristics (which is the case for the first experiment), as it was done in previous studies (Kuhnen and Knutson, 2005). Adopting a stricter random-effects approach, we next calculated the subjectspecific coefficients of the logistic regression and then evaluated these parameters in second-level random-effects tests.

For the first experiment, striatal and dACC responses were significant $(p<$ $0.001)$, whereas IFG approached significance $(p=0.10)$. Yet, it should be remembered that the BOLD response of the IFG is more relevant as risk aversion increases. Therefore, if we only include participants that are at least slightly risk averse (i.e., the difference between the CEs is $\geq 3$ monetary units, which is $1 \mathrm{SD}$ away from the average value of the sample), then the IFG response indeed becomes significant at $p=0.05$. This implies that the IFG BOLD response plays a role in the forthcoming choice primarily when the participant is risk averse.

To test this notion further, we performed a similar randomeffects analysis also in the second experiment. In agreement with the findings from the first experiment, the significance of the IFG BOLD response for the logistic regression model increases when we exclude risk-neutral participants (responses of the three structures are all significant at $p=0.05$ ). These data further reinforce the notion that dACC, IFG, and striatal BOLD responses contribute to decisions in risky situations, with IFG response being more relevant to the decisions of risk-averse agents.

Using the coefficients, we determined the model-based computed probability of a risky choice, given the activity of VSt, dACC, and IFG on each trial. We then calculated the receiver operating characteristic (ROC) curve comparing these modelbased probabilities of a risky choice with the actual choice. The ROC analysis describes how effectively an ideal observer would detect a signal (a risky choice, in our case) in the presence of noise. The ROC has been effectively used (Britten et al., 1996; Chandrasekaran et al., 2007; Thielscher and Pessoa, 2007) to elucidate the relation between neuronal responses and perceptual choices.

For the choice trials of the first experiment, the model detected the behavioral choice significantly well above chance $(\mathrm{ROC}=0.77$; $p<0.01$ ) (Fig. 4A). We used exactly the same method in the second experiment and again found a similar $\mathrm{ROC}$ value $(\mathrm{ROC}=0.74 ; p<$ 0.01 ) (Fig. $4 A$ ). In addition, the ROC values derived from models using each structure separately were lower (Fig. $4 A$ ). In the nochoice trials, the ROC value was also lower $(\mathrm{ROC}=0.72)$.

Stricter validation analyses confirmed the generalizability of the detective power of the model. Specifically, leave-one-out cross-validation produced a similar, statistically significant ROC value $(\mathrm{ROC}=0.65)$, which was slightly lower because of the smaller sample size inherent in the procedure.

\section{Discussion}

From both a behavioral and theoretical perspective, the value and risk of an option along with the agent's risk aversion are the basic 
factors implicated in the choice behavior. Our approach in studying choice behavior was to first identify the components of the system (magnitude, risk, and risk aversion) and then to piece them together to produce a function relating them to a behavioral outcome (i.e., the choice). To achieve this, we first located the neuronal responses that are more relevant to decision factors. In the final step, we tested whether these responses can indeed describe the function of the system (i.e., detect the behavioral choice).

Previous studies have uncovered the neural correlates of independent decision factors as well. Our design disentangled decision parameters from utility encoding, took into account the behaviorally demonstrated risk attitudes of each participant, and minimized any learning elements. The present findings suggest that the computational and theoretical deconstruction of the decision procedure into specific parameters meets with distinct BOLD responses, which could contribute crucial inputs for actual choices.

In two different experiments, in which important behavioral parameters were differentiated, we found distinct neuronal responses toward different decision factors. The striatum was particularly responsive to changes in magnitude, dACC was involved in mainly objective risk coding, and IFG signaled risk aversion. Importantly, by combining the information from these different brain regions, these BOLD responses were informative enough to allow an ideal observer to detect the overt choice: a risky choice was more probable when striatal and cingulate activity was higher, whereas increased BOLD signals from IFG correlated with increased probability of a safe choice.

High correlations between BOLD responses and personality traits have been criticized recently (Vul et al., 2009). Although the critique is highly controversial and disputable (Lieberman et al., 2009), our study nevertheless escapes the criticism because we use two separate sets of data (and experimental designs) to evaluate our hypotheses; in both experiments, the BOLD response of the brain regions correlated with the behavioral measurement. In addition, the principal measurement (risk aversion) is not approached as a personality trait but rather as a behavioral measurement. Finally, the regions reflecting individual differences in risk processing were identified independently from those coding risk.

\section{Value, objective, and subjective risk}

Our results show that VSt activation increases with increasing value. Importantly, our analysis suggests that striatal activity encoded value, independent from utility. Because of our design, the term "value" refers to either "magnitude" (first experiment) or "expected value" (second experiment). We need to underline that our experiments do not clarify to which of these two parameters the VSt is responsive to, as such a study would require a design that includes different levels of probabilities. This should be addressed in future studies, although similar issues have been tested by previous reports; our results are in line with their results linking striatal activity to computing value (or processing its components) (Knutson et al., 2005; Abler et al., 2006; Yacubian et al., 2006; Tobler et al., 2007).

According to the present results, dACC activity increases when the forthcoming choice has higher risk. The magnitude of this increase does not covary with individual differences in the estimation of risk (risk aversion). Therefore, dACC activity seems to preferentially mirror an objective metric of risk. In addition, we found no differences in dACC activity with respect to the utility of the subsequent choice. Previous studies have implicated dACC activity with the volatility of the reward environment
(Behrens et al., 2007), whereas Critchley et al. (2001) relate the increased BOLD response of ACC in anticipation of risky outcomes to autonomic arousal. We also control for conflict of choice (Carter et al., 1998), which is a common function ascribed to ACC, because both alternatives are equally preferred; therefore, conflict in every trial of the first experiment is maximal. A possible caveat of our study is that both experiments have a relatively small number of participants; this might significantly lower the power to find correlations. Although the size of our sample was sufficient to detect correlations in IFG, the fact that we did not find a correlation of dACC BOLD response to risk does not necessarily preclude the possibility that this area might also be sensitive in subject-wise differences in risk assessments.

However, it should be emphasized that the brain responses attributed to specific decision parameters are not exclusive but mainly preferential. Our study adopted a formal definition of risk, which is independent of changes in probabilities; this is a crucially different aspect of risk (Rushworth and Behrens, 2008). The control of probability might be a contributing factor for not finding risk signals in brain structures such as insula (Critchley et al., 2001; Preuschoff et al., 2008) and areas of prefrontal cortex (Elliott et al., 1999; Rogers et al., 1999), yet a thorough examination of risk-related choice behavior necessitates the detailed, separate identification of the different facets of risk. Given that variance is the first moment of a distribution, it is evident that it is one of the primary aspects of risk.

IFG BOLD responses found in this study reflected risk aversion. The currently observed BOLD response of IFG is located within right dorsolateral prefrontal cortex whose stimulation accordingly modulates risk aversion (Knoch et al., 2006; Fecteau et al., 2007). Our results demonstrate that this area does not influence the objective evaluation of risk but rather the subjective perception of the riskiness of the option. Additional analysis suggests that this IFG BOLD response functions as a "safety" signal, because it shows higher response to safer options, especially for more risk-averse participants.

\section{Combined BOLD signals contributing to decision making}

To use an analogy, in perceptual decisions, the choice can be decoded by comparing neuronal activity between areas that are selectively tuned to the basic characteristics of each option [for instance, areas sensitive to either faces or houses (Heekeren et al., 2004)]. Lee et al. (2007) suggest that, to make a choice, the brain should collect information on different decision parameters and then combine this information in an effective way to produce the choice. In economic choices, specific values are assigned to the individual options; these values are modulated, among others, by the risk of the options. The conjecture that risk has an influence on value constitutes the key characteristic of one of the prominent theories in economic decision making, namely the mean variance approach (Levy and Markowitz, 1979; Preuschoff et al., 2006; Rangel et al., 2008). Essentially, the underlying hypothesis is that the overt choice is the output of internal processes combining the neuronal information pertaining to each choice parameter. Our experiment followed this rationale of combined decision parameters.

We indeed found a group of areas that are sensitive to specific decision parameters. Logistic regression analysis of signals from different regions revealed relationships not obvious from singlestructure analysis. The relationship between activity and choice can be approximated by a competing activity between striatum and AACC on one hand, correlating with riskier choices, and IFG on the other hand, holding an inhibitory, risk-averse role. 
Our analysis brings forward the possibility of evaluating the effect of "virtual" lesions in the implicated areas. Striatal and cingulate lesions would potentially be associated with less riskaverse (and more risk neutral) choices. A striatal lesion could reduce the ability to evaluate magnitude, an effect that is also implied by negative motivational changes in patients with globus pallidus lesions (Vijayaraghavan et al., 2008). Nevertheless, such a lesion could be compensated by functions in other areas, namely ventromedial prefrontal cortex. In addition, our prediction is that lateral prefrontal cortex lesions will lead to riskier choices, which, as mentioned previously, is in accordance with neuromodulatory studies (Knoch et al., 2006; Fecteau et al., 2007). A recent study (Gianotti et al., 2009) also suggested that participants with higher baseline cortical activity in the right prefrontal cortex are more risk averse. In addition, patients with predominantly right-sided prefrontal lesions demonstrate a riskier behavior (Clark et al., 2003).

It should be noted that individual brain regions, and especially striatum, independently have high ROC values. The latter suggests that encoding of isolated decision parameters already contains information able to decode the choice, yet the incorporation and appropriate combination of information stemming from aptly selected regions improves the overall representation of the choice behavior.

Cognitive functions such as decision making might necessitate the combination of signals from different brain areas instead of contributions from a single structure. Such distributed neuronal contributions to cognitive functions have also been found in other paradigms, such as emotional perceptual decisions (Pessoa and Padmala, 2005) and a probabilistic-reversal learning task (Hampton and O'Doherty, 2007). Our study demonstrates that neural combinations of information can be beneficial on economic decisions under risk, as well.

It has been suggested (MacDonald et al., 2000; Fleck et al., 2006) that dACC engagement indexes conflict and the need for cognitive control (Barch et al., 2001), whereas DLPFC assumes a more evaluative role, including cognitive control and response selection. Importantly, Rushworth et al. (2004) suggest that the main function of ACC is to perform a cost-benefit analysis to guide action. The present results fit in that framework. Dorsal ACC evaluates the riskiness of the situation (which may correspond to an evaluation of costs and benefits), indexing the need to engage cognitive control over the competing choice between a risky and a safe alternative. Higher risk requires higher cognitive control compared with low risk trials. Therefore, dorsal ACC activity signals whether and to what extent cognitive control is needed according to the riskiness of the situation, whereas IFG/DLPFC activity idiosyncratically guides the choice according to risk attitudes.

In conclusion, our analysis sheds light on the mechanisms used in decision making under risk. Behavioral evidence suggests that the output of the choice process heavily depends on the statistical properties of the options. This implies that the brain not only encodes these properties but also combines them to produce the overt choice. An analogous mechanism is suggested by our data. From a more general point of view, the generation and combination of neuronal signals representing lower-level properties of the stimulus might be a general decision-making mechanism across different modalities (Heekeren et al., 2008).

\section{References}

Abler B, Walter H, Erk S, Kammerer H, Spitzer M (2006) Prediction error as a linear function of reward probability is coded in human nucleus accumbens. Neuroimage 31:790-795.
Barch DM, Braver TS, Akbudak E, Conturo T, Ollinger J, Snyder A (2001) Anterior cingulate cortex and response conflict: effects of response modality and processing domain. Cereb Cortex 11:837-848.

Behrens TE, Woolrich MW, Walton ME, Rushworth MF (2007) Learning the value of information in an uncertain world. Nat Neurosci 10:12141221.

Binswanger HP (1980) Attitudes toward risk: experimental measurement in rural India. Am J Agric Econ 62:395-407.

Breiter HC, Aharon I, Kahneman D, Dale A, Shizgal P (2001) Functional imaging of neural responses to expectancy and experience of monetary gains and losses. Neuron 30:619-639.

Britten KH, Newsome WT, Shadlen MN, Celebrini S, Movshon JA (1996) A relationship between behavioral choice and the visual responses of neurons in macaque MT. Vis Neurosci 13:87-100.

Brown JW, Braver TS (2005) Learned predictions of error likelihood in the anterior cingulate cortex. Science 307:1118-1121.

Brown JW, Braver TS (2008) A computational model of risk, conflict, and individual difference effects in the anterior cingulate cortex. Brain Res 1202:99-108.

Carter CS, Braver TS, Barch DM, Botvinick MM, Noll D, Cohen JD (1998) Anterior cingulate cortex, error detection, and on-line monitoring of performance. Science 280:747-749.

Chandrasekaran C, Canon V, Dahmen JC, Kourtzi Z, Welchman AE (2007) Neural correlates of disparity-defined shape discrimination in the human brain. J Neurophysiol 97:1553-1565.

Clark L, Manes F, Antoun N, Sahakian BJ, Robbins TW (2003) The contributions of lesion laterality and lesion volume to decision-making impairment following frontal lobe damage. Neuropsychologia 41:1474-1483.

Critchley HD, Mathias CJ, Dolan RJ (2001) Neural activity in the human brain relating to uncertainty and arousal during reward anticipation. Neuron 29:537-545.

De Martino B, Kumaran D, Seymour B, Dolan RJ (2006) Frames, biases, and rational decision-making in the human brain. Science 313:684-687.

Dale AM (1999) Optimal experimental design for event-related fMRI. Hum Brain Mapp 8:109-114.

Dohmen T, Falk A, Huffman D, Sunde U, Schupp J, Wagner G (2005) Individual risk attitudes: new evidence from a large, representative, experimentallyvalidated survey. IZA DP No. 1730.

Elliott R, Rees G, Dolan RJ (1999) Ventromedial prefrontal cortex mediates guessing. Neuropsychologia 37:403-411.

Fecteau S, Pascual-Leone A, Zald DH, Liguori P, Théoret H, Boggio PS, Fregni F (2007) Activation of prefrontal cortex by transcranial direct current stimulation reduces appetite for risk during ambiguous decision making. J Neurosci 27:6212-6218.

Fleck MS, Daselaar SM, Dobbins IG, Cabeza R (2006) Role of prefrontal and anterior cingulate regions in decision-making processes shared by memory and nonmemory tasks. Cereb Cortex 16:1623-1630.

Friston KJ, Holmes AP, Worsley KJ, Poline J-B, Frith C, Frackowiak RSJ (1994) Statistical parametric maps in functional brain imaging: a general linear approach. Hum Brain Mapp 2:189-210.

Friston KJ, Holmes AP, Worsley KJ (1999) How many subjects constitute a study? Neuroimage 10:1-5.

Gianotti LR, Knoch D, Faber PL, Lehmann D, Pascual-Marqui RD, Diezi C, Schoch C, Eisenegger C, Fehr E (2009) Tonic activity level in the right prefrontal cortex predicts individuals' risk taking. Psychol Sci 20:33-38.

Glaser D, Friston KJ (2003) Variance components. In: Human brain function, Ed 2 (Frackowiak RSJ, Friston KJ, Frith CD, Dolan RJ, Price CJ, Ashburner JT, Penny WD, Zeki S, eds). San Diego: Academic.

Hampton AN, O'Doherty JP (2007) Decoding the neural substrates of reward-related decision making with functional MRI. Proc Natl Acad Sci U S A 104:1377-1382.

Heekeren HR, Marrett S, Bandettini PA, Ungerleider LG (2004) A general mechanism for perceptual decision making in the human brain. Nature 431:859-862.

Heekeren HR, Marrett S, Ungerleider LG (2008) The neural systems that mediate human perceptual decision making. Nat Rev Neurosci 9:467-479.

Holt C, Laury KS (2002) Risk aversion and incentive effects in lottery choices. Am Econ Rev 92:1644-1655.

Hosmer D, Lemeshow S (1989) Applied logistic regression. New York: Wiley.

Huettel SA, Stowe CJ, Gordon EM, Warner BT, Platt ML (2006) Neural 
signatures of economic preferences for risk and ambiguity. Neuron 49: 765-775.

Kahneman D, Wakker P, Sarin R (1997) Back to Bentham? Explorations of experienced utility. Q J Econ 112:375-406.

Knoch D, Gianotti LR, Pascual-Leone A, Treyer V, Regard M, Hohmann M, Brugger P (2006) Disruption of right prefrontal cortex by low-frequency repetitive transcranial magnetic stimulation induces risk-taking behavior. J Neurosci 26:6469-6472.

Knutson B, Bossaerts P (2007) Neural antecedents of financial decisions. J Neurosci 27:8174-8177.

Knutson B, Adams CM, Fong GW, Hommer D (2001) Anticipation of increasing monetary reward selectively recruits nucleus accumbens. J Neurosci 21:RC159(1-5).

Knutson B, Taylor J, Kaufman M, Peterson R, Glover G (2005) Distributed neural representation of expected value. J Neurosci 25:4806-4812.

Kuhnen CM, Knutson B (2005) The neural basis of financial risk taking. Neuron 47:763-770.

Lee D, Rushworth MF, Walton ME, Watanabe M, Sakagami M (2007) Functional specialization of the primate frontal cortex during decision making. J Neurosci 27:8170-8173.

Levy H, Markowitz HM (1979) Approximating expected utility by a function of mean and variance. Am Econ Rev 69:308-317.

Lieberman MD, Berkman ET, Wager TD (2009) Correlations in social neuroscience aren't voodoo: commentary on Vul et al. (2009). Perspect Psychol Sci 4:299-307.

Lohrenz T, McCabe K, Camerer CF, Montague PR (2007) Neural signature of fictive learning signals in a sequential investment task. Proc Natl Acad Sci U S A 104:9493-9498.

Loomes G, Sugden R (1982) Regret theory: an alternative theory of rational choice under uncertainty. Econ J R Econ Soc 92:805-824.

Luce R (2000) Utility of gains and losses: measurement-theoretical and experimental approaches. Mahwah, NJ: Erlbaum.

MacDonald AW 3rd, Cohen JD, Stenger VA, Carter CS (2000) Dissociating the role of the dorsolateral prefrontal and anterior cingulate cortex in cognitive control. Science 288:1835-1838.

Maldjian JA, Schulder M, Liu WC, Mun IK, Hirschorn D, Murthy R, Carmel P, Kalnin A (1997) Intraoperative functional MRI using a real-time neurosurgical navigation system. J Comput Assist Tomogr 21:910-912.

Martinez D, Slifstein M, Broft A, Mawlawi O, Hwang DR, Huang Y, Cooper T, Kegeles L, Zarahn E, Abi-Dargham A, Haber SN, Laruelle M (2003) Imaging human mesolimbic dopamine transmission with positron emission tomography. Part II: amphetamine-induced dopamine release in the functional subdivisions of the striatum. J Cereb Blood Flow Metab 23: 285-300.

McCoy AN, Platt ML (2005) Risk-sensitive neurons in macaque posterior cingulate cortex. Nat Neurosci 8:1220-1227.

Murray GK, Corlett PR, Clark L, Pessiglione M, Blackwell AD, Honey G, Jones PB, Bullmore ET, Robbins TW, Fletcher PC (2008) Substantia nigra/ventral tegmental reward prediction error disruption in psychosis. Mol Psychiatry 13:239, 267-276.
Paulus MP, Rogalsky C, Simmons A, Feinstein JS, Stein MB (2003) Increased activation in the right insula during risk-taking decision making is related to harm avoidance and neuroticism. Neuroimage 19:1439-1448.

Pessoa L, Padmala S (2005) Quantitative prediction of perceptual decisions during near-threshold fear detection. Proc Natl Acad Sci USA 102:5612-5617.

Preuschoff K, Bossaerts P, Quartz SR (2006) Neural differentiation of expected reward and risk in human subcortical structures. Neuron 51:381-390.

Preuschoff K, Quartz SR, Bossaerts P (2008) Human insula activation reflects risk prediction errors as well as risk. J Neurosci 28:2745-2752.

Rangel A, Camerer C, Montague PR (2008) A framework for studying the neurobiology of value-based decision making. Nat Rev Neurosci 9:545-556.

Rogers RD, Owen AM, Middleton HC, Williams EJ, Pickard JD, Sahakian BJ, Robbins TW (1999) Choosing between small, likely rewards and large, unlikely rewards activates inferior and orbital prefrontal cortex. J Neurosci 19:9029-9038.

Rolls ET, McCabe C, Redoute J (2008) Expected value, reward outcome, and temporal difference error representations in a probabilistic decision task. Cereb Cortex 18:652-663.

Rorden C, Brett M (2000) Stereotaxic display of brain lesions. Behav Neurol 12:191-200.

Rothschild M, Stiglitz J (1970) Increasing risk: a definition. J Econ Theory 2:225-243.

Rushworth MF, Behrens TEJ (2008) Choice, uncertainty and value in prefrontal and cingulate cortex. Nat Neurosci 11:389-397.

Rushworth MF, Walton ME, Kennerley SW, Bannerman DM (2004) Action sets and decisions in the medial frontal cortex. Trends Cogn Sci 8:410-417.

Thielscher A, Pessoa L (2007) Neural correlates of perceptual choice and decision making during fear-disgust discrimination. J Neurosci 27:2908-2917.

Tobler PN, O'Doherty JP, Dolan RJ, Schultz W (2007) Reward value coding distinct from risk attitude-related uncertainty coding in human reward systems. J Neurophysiol 97:1621-1632.

Vijayaraghavan L, Vaidya JG, Humphreys CT, Beglinger LJ, Paradiso S. (2008) Emotional and motivational changes after bilateral lesions of the globus pallidus. Neuropsychology 22:412-418.

Vul E, Harris C, Winkielman P, Pashler H (2009) Puzzlingly high correlations in fMRI studies of emotion, personality, and social cognition. Perspect Psychol Sci 4:274-290.

Weber EU, Shafir S, Blais AR (2004) Predicting risk sensitivity in humans and lower animals: risk as variance or coefficient of variation. Psychol Rev 111:430-445.

Worsley KJ, Marrett S, Neelin P, Vandal AC, Friston KJ, Evans AC (1996) A unified statistical approach for determining significant signals in images of cerebral activation. Hum Brain Mapp 4:58-73.

Yacubian J, Gläscher J, Schroeder K, Sommer T, Braus DF, Büchel C (2006) Dissociable systems for gain- and loss-related value predictions and errors of prediction in the human brain. J Neurosci 26:9530-9537. 